Anaesthesist 2021 · 70:93-96

https://doi.org/10.1007/s00101-020-00887-3

(C) Springer Medizin Verlag GmbH, ein Teil von Springer Nature 2020
Vera von Dossow ${ }^{1} \cdot$ Bernhard Zwißler $^{2}$

'Institut für Anästhesiologie, Herz- und Diabeteszentrum NRW, Universitätsklinik, Ruhr-Universität Bochum, Bad Oeynhausen, Deutschland

${ }^{2}$ München, Deutschland

\title{
Minimal-invasive Klappeninterventionen durch ein multidisziplinäres Team
}

\section{Sichere und erfolgreiche Patientenbehandlung}

Der Begriff „minimal-invasive Klappeninterventionen“ bei Herzklappenerkrankungen beinhaltet die kathetergestützten Implantationen von Herzklappen und die perkutanen kathetergestützten Verfahren zur Reduktion von Klappeninsuffizienzen (Mitraclip/Trikuspidal-Clip). In den letzten 10 Jahren haben diese Verfahren in der Herzmedizin einen Paradigmenwechsel hervorgerufen. Die Zahl minimal-invasiver Klappeninterventionen in Europa und in Deutschland nimmt dynamisch zu $[1,2]$.

Vigilius-Rauch et al. legen mit dem in diesem Heft von Der Anaesthesist publizierten Beitrag [13] eine hervorragende Übersichtsarbeit vor, die alle relevanten Aspekte rund um die anästhesiologische Versorgung minimal-invasiver Klappeninterventionen abdeckt und den Lesern einen klinisch und wissenschaftlich fundierten Leitfaden für das Management dieser trotz der minimalen Invasivität hochkomplexen Eingriffe gibt.

Mittlerweile hat die kathetergestützte Aortenklappenimplantation (TAVIProzedur) als häufigste Klappenintervention einen hohen Stellenwert in der klinischen Versorgung in Deutschland. Große randomisierte Studien konnten nachweisen [3], dass nicht nur Patienten mit einem hohen Operationsrisiko bzw. für einen offenen herzchirurgischen Eingriff ungeeignete Patienten signifikant von diesem Verfahren profitieren, sondern auch ältere Patienten mit einem mittleren (Society of Thoracic
Surgeons Score [STS] $<8 \%$ ) oder geringen (STS $<4 \%$ ) operativen Risiko. Die erst kürzlich (März 2020) publizierten Konsensusempfehlungen der Deutschen Gesellschaft für Kardiologie (DGK) und der Deutschen Gesellschaft für Thorax-, Herz- und Gefäßchirurgie (DGTHG) zur kathetergestützten TAVI-Implantation fassen in einer Übersicht die aktuelle evidenzbasierte Datenlage hervorragend zusammen [2]. In der PARTNER-3Studie („Placement of Aortic Transcatheter Valves") wurden 1000 Patienten mit einem mittleren Alter von 73 Jahren und einem STS Score von $1,9 \%$ für eine TAVI oder einen konventionellen Aortenklappenersatz (AKE) randomisiert $[4,5]$ : Der primär definierte Endpunkt (Todesrate, Schlaganfall und erneute Hospitalisierung nach einem Jahr) trat in der TAVI-Gruppe signifikant seltener auf als nach AKE. Das „Evolut Low Risk Trial“ an 1468 Patienten zeigte hinsichtlich des primären Endpunktes (Kombination von Tod und behinderndem Schlaganfall nach 2 Jahren) keine Unterlegenheit der TAVI im Vergleich zur AKE [6]. In Anlehnung an diese Daten erfolgte zudem eine Datenanalyse aus dem German Aortic Valve Registry (GARY) bei Patienten mit einem niedrigen Operationsrisiko (STS <4\%; [7]). Hierbei zeigen sich in einem "propensity score matching " an einer Gesamtzahl von 20.549 Patienten >70 Jahre eine höhere Krankenhaus- sowie eine höhere Dreißigtageüberlebensrate der TAVI im 
Vergleich zu AKE-Patienten. Allerdings konnte dieser Unterschied nach einem Jahr nicht mehr nachgewiesen werden. Für jüngere Patienten $<70$ Jahre liegen zum jetzigen Zeitpunkt noch keine ausreichenden endgültigen konklusiven Registerdaten vor.

Neben den Aortenklappenerkrankungen stehen die Mitralklappeninsuffizienz an zweiter und die Trikuspidalinsuffizienz an dritter Stelle der Herzklappenerkrankungen [8]. Somit ist es nicht verwunderlich, dass die kathetergestützten Therapien dieser Klappeninsuffizienzen in Deutschland ebenfalls einen zunehmenden Stellenwert in der klinischen Versorgung hochbetagter und multimorbider Patienten einnehmen. Ziel ist eine signifikante Reduktion der Insuffizienz unter Vermeidung einer hämodynamisch wirksamen Stenose. Ein wichtiger Aspekt der Prozedur ist die Tatsache, dass die Intervention am schlagenden Herzen durchgeführt wird und das postinterventionelle Ergebnis von intrakardialen Füllungsdrücken beeinflusst werden kann. Die transösophageale Echokardiographie (TEE) erlaubt hier die kontinuierliche Visualisierung der Klappenfunktion, des Füllungszustands und der Kontraktilität des Herzens. Die transthorakale Echokardiographie (TTE) und die TEE stellen hier unverzichtbare Instrumente im periinterventionellen Setting dar.

Kontrollierte randomisierte Studien für das MitraClip-Verfahren (Fa. Abbott Vascular, Abbott Park, IL, USA) mit Langzeitdaten liegen bislang nicht vor: In der Everest-II-Studie zeigte sich für das MitraClip-Verfahren zwar ein signifikant erhöhtes Risiko für einen erneuten Eingriff im Vergleich zum operativen Prozedere, allerdings mit weniger Blutungskomplikationen [9]. Die Autoren schlussfolgerten, dass es sich um ein sicheres Verfahren handelt. Auch zeigen die Daten des Deutschen Mitralklappenregisters (Transcatheter Mitral Valve Interventions [TRAMI] Register) hohe Erfolgs- bei geringen Komplikationsraten [9]. Auch für das Anuloplastieverfahren, das CE-zertifizierte Cardioband, konnte an einem kleinen Patientenkollektiv eine $>80 \%$ ige Erfolgsrate nachgewiesen werden [10].

Die kathetergestützten Verfahren zur Reduktion der Trikuspidalinsuffizienz befinden sich aktuell in der Entwicklung, allerdings zeigt sich bereits ein sehr dynamischer Trend, sodass in absehbarer Zeit auch kontrollierte und randomisierte Studien erste Langzeitergebnisse zeigen werden $[1,8]$.

\section{》) Die Betreuung der betroffenen Patienten durch ein Heart-Team ist eine weitsichtige Entscheidung}

Die interdisziplinäre Zusammenarbeit in Form des „Heart-Teams" entspricht der im Juli 2015 formulierten Gesetzgebung des gemeinsamen Bundesausschusses. Die Entscheidung, minimal-invasive Klappeninterventionen in einem multidisziplinären Team zu betreuen, ist eine Entscheidung mit Weitsicht. Sie entspricht den demografischen Herausforderungen, denen wir uns zunehmend stellen müssen. Der dritte Partner im „Heart-Team“ ist die Herzanästhesie. Die komplexen Techniken benötigen speziell geschulte Anästhesieteams. Die Richtlinien zur Weiterbildung in der TTE und TEE für Anästhesisten durch die Deutsche Gesellschaft für Anästhesiologie und Intensivmedizin (DGAI) bilden eine erste Grundlage für die Weiterentwicklung des bisherigen Wissens. So stellt beispielsweise die 3DTEE eine weitere Herausforderung an den in der Herzanästhesie erfahrenen Anästhesisten dar, gibt ihm aber auch zugleich die Möglichkeit, seine Kompetenzen zu erweitern und von der engen interdisziplinären Zusammenarbeit mit den Kardiologen und Herzchirurgen zu profitieren.

Durch die enge Kooperation aller 3 Teams können wir unseren Patienten innovative Herzmedizin unter höchsten Qualitäts- und Sicherheitsstandards anbieten. Die Aufgaben des Herz-Teams in den kürzlich publizierten Konsensusempfehlungen der DGK und der DGTHG sind klar definiert: Zusätzlich zum Entscheidungsprozess sollen Risikofaktoren wie Gebrechlichkeit, Multimorbidität und auch der Patienten- wunsch berücksichtigt werden [2]. Das hochbetagte und multimorbide Patientenkollektiv stellt für jede Fachdisziplin eine Herausforderung dar. Gerade bei diesen Patienten bedarf es einer engen interdisziplinären Abstimmung für eine patientenindividuelle Behandlung, um Patientensicherheit auf höchstem Niveau zu gewährleisten. Diese ist genauso wichtig wie maximale hämodynamische Stabilität und optimaler Patientenkomfort. Alle 3 genannten Faktoren tragen $\mathrm{zu}$ einem effektiven Ergebnis der Intervention und auch zu einer raschen postinterventionellen Erholung bei. Nicht zuletzt findet der Begriff Gebrechlichkeit (,frailty“) auch im Rahmen der interventionellen Klappeninterventionen Einzug in die europäischen Leitlinien zur Behandlung von Aortenklappenerkrankungen und ist sogar im Vergleich mit den üblichen verwendeten konventionellen Risiko-Scores wie dem STS oder Euro-SCORE in der Prädiktion des Ergebnisses überlegen [11].

\section{Wahl der periinterventionellen Betreuung}

Die Wahl des Anästhesieverfahrens ist eine patientenindividualisierte Entscheidung, die im Vorfeld mit dem Patienten gemeinsam durch eine sorgfältige Aufklärung und Risikostratifizierung erörtert wird. Hierbei geht es nicht nur um die Entscheidung Allgemeinanästhesie, Analgosedierung oder lediglich „Anästhesie-Stand-by“ mit örtlicher Betäubung. Auch sollte die Wahl des Anästhesieverfahrens nicht in Abhängigkeit von der Erfahrung des Interventionsteams und den prozeduralen Prozesszeiten getroffen werden. Wichtig ist es zu betonen, dass ein in der Herzanästhesie erfahrener Anästhesist auf höchstem Niveau zu jedem Zeitpunkt eine patientensichere Betreuung gewährleistet. Nicht zwangsläufig ist hier nur die Allgemeinanästhesie mit einer Intubation des Patienten gemeint. Differenzierte Konzepte zur Fast-Track-Anästhesie und Analgosedierung, die evidenzbasiert im Rahmen von „standard operating procedures“ in den letzten Jahren weiterentwickelt wurden, sorgen heutzutage für maximale Patientensicherheit und optimalen Patienten- 
Hier steht eine Anzeige.

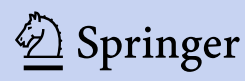


komfort. Bei der Auswahl der Medikationen stehen Stressreduktion, adäquate Analgesie und optimale Bedingungen für das interventionelle „Heart-Team“ („ruhig schlafender, erweckbarer und nichtagitierter Patient, keine spontanen Bewegungen, kein Husten bei erhaltenem Aspirationsschutz") im Vordergrund.

Vereinzelt gibt es Patienten, bei dem das periinterventionelle Betreuungsverfahren umgestellt und angepasst werden muss (z.B. keine Toleranz flacher Lagerung bei respiratorischer Insuffizienz). Sehr selten muss in solchen Fällen eine Atemwegssicherung mithilfe der Larynxmaske oder eine Intubationsnarkose durchgeführt werden. Gelegentlich ist es sinnvoll, bereits im Vorfeld eine Intubation zu planen. Vor allem Patienten mit einer stark eingeschränkten Herzfunktion und respiratorischen Insuffizienz profitieren in solchen Fällen von einer Allgemeinanästhesie, da diese eine sehr geringe Toleranz für hämodynamische Instabilitäten im Rahmen des „rapid pacing“ bei Implantation der Aortenklappe aufweisen, v.a. dann, wenn diese Maßnahme während der TAVI-Prozedur mehrfach wiederholt werden muss. Auch im Rahmen von Gefäßkomplikationen ist gelegentlich ein Konvertieren auf eine Allgemeinanästhesie erforderlich. Vor allem aber auch bei längeren Interventionszeiten in strenger Rückenlage klagen die Patienten sehr häufig über Rückenschmerzen, was ebenfalls $\mathrm{zu}$ einer Vertiefung des Analgosedierungsgrades in Richtung Allgemeinanästhesie führen kann. Bei der Beantwortung der Frage, welches Anästhesieverfahren das bevorzugte bei der TAVI-Implantation ist, existieren bislang sehr kontroverse Diskussionen zwischen den Vertretern der internationalen Anästhesie-Fachgesellschaften. Während weltweit das Verfahren der Allgemeinanästhesie noch überwiegend favorisiert wird, hat sich in Europa und Deutschland der Trend zu einer Analgosedierung oder auch „flachen Sedierung“ mit Lokalanästhesie an den meisten großen TAVI-Zentren durchgesetzt. Mit der kürzlich in der internationalen Fachzeitschrift Circulation publizierten Studie „The randomized TAVI-SOLVE-Trial“ konnten die Autoren Thiele et al. erst- malig für das Konzept Analgosedierung vergleichbare Ergebnisse in Bezug auf Krankenhaussterblichkeit, Schlaganfallund Myokardinfarktrate zeigen [12]. Die Analgosedierung war dem Verfahren einer Allgemeinanästhesie nicht unterlegen. Die Autoren schlussfolgerten, dass eine Analgosedierung bei der kathetergestützten TAVI-Implantation vergleichbar patientensicher ist. Allerdings bleibt die Frage offen, ob zur Bewertung eines Anästhesieverfahrens tatsächlich die in dieser Studie gewählten primären Endpunkte die geeigneten sind.

\section{Fazit}

Die unterschiedlichen Techniken der minimal-invasiven Klappeninterventionen erweitern das Spektrum der „klassischen Herzanästhesie“ und stellen neue Anforderungen an den in der Herzanästhesie erfahrenen Anästhesisten. Es setzt ein hohes Maß an interdisziplinärer Abstimmung und interprofessioneller Kommunikation voraus, um maximale Patientensicherheit und Therapieerfolg für dieses Hochrisikokollektiv zu garantieren.

Unbestritten ist die Chance und bleibt somit auch die Herausforderung für die Zukunft, gemeinsam als multidisziplinäres Team zu agieren.

\section{Korrespondenzadresse}

\section{Univ.-Prof. Dr. Vera von Dossow}

Institut für Anästhesiologie, Herz- und

Diabeteszentrum NRW, Universitätsklinik, Ruhr-Universität Bochum

Georgstr. 11, 32545 Bad Oeynhausen,

Deutschland

vvondossow@hdz-nrw.de

Interessenkonflikt. V. von Dossow und B. Zwißler geben an, dass kein Interessenkonflikt besteht.

\section{Literatur}

1. Baldus $S$ et al (2020) Interventional treatment of AV valvular diseases-Criteria for certification of mitral valve centers Position paper of the German Cardiac Society. Kardiologe 14(5):339-363

2. Kuck KH et al (2020) Consensus paper of the German Cardiac Society (DGK) and the German Society for Thoracic and Cardiovascular Surgery (DGTHG) on transcatheter aortic valve implantation (TAVI) 2020. Kardiologe 14(3):182-204
3. Bleiziffer $\mathrm{S}$ et al (2020) Long-term outcomes after transcatheter aortic valve implantation in failed bioprosthetic valves. Eur Heart J 41(29):2731-2742

4. Vemulapalli S, Carroll JD, Mack MJ (2019) Volume and outcomes for transcatheter aortic-valve replacement reply. N Engl J Med 381(14):1394-1395

5. Mack MJ et al (2019) Transcatheter aortic-valve replacement with a balloon-expandable valve in low-risk patients. N Engl J Med 380(18):1695-1705

6. Popma JJ et al (2019) Transcatheter aortic-valve replacement with a self-expanding valve in lowrisk patients. N Engl J Med 380(18):1706-1715

7. Bekeredjian R et al (2019) Patients at low surgical risk as defined by the Society of Thoracic Surgeons Score undergoing isolated interventional or surgical aortic valve implantation: in-hospital data and 1-year results from the German Aortic Valve Registry (GARY). Eur Heart J40(17):1323-1330

8. Baldus S et al (2018) Interventional therapy for AV valve disease-focus onmitral valve regurgitation. Position paper of the German Cardiac Society. Kardiologe 12(2):128-144

9. Feldman T et al (2011) Percutaneous repair or surgery for mitral regurgitation. $\mathrm{N}$ Engl J Med 364(15):1395-1406

10. Maisano F et al (2016) Cardioband, a transcatheter surgical-like direct mitral valve annuloplasty system: early results of the feasibility trial. Eur Heart J37(10):817-825

11. Assmann P et al (2016) Frailty is associated with delirium and mortality after transcatheter aortic valve implantation. Open Heart 3(2):e478

12. Thiele Hetal (2020) General versus local anesthesia with conscious sedation in transcatheter aortic valve implantation: the randomized SOLVE-TAVI trial. Circulation 142(15):1437-1447

13. Vigelius-Rauch U, Zajonz T, Sander M (2020) Anästhesiologische Implikationen bei minimalinvasiven Klappeninterventionen. Anaesthesist. https://doi.org/10.1007/s00101-020-00847-x 